\title{
Serine/threonine protein phosphatase 6 modulates the radiation sensitivity of glioblastoma
}

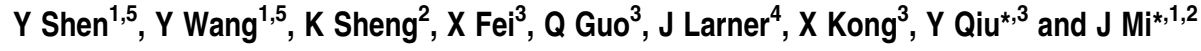

Increasing the sensitivity of glioblastoma cells to radiation is a promising approach to improve survival in patients with glioblastoma multiforme (GBM). This study aims to determine if serine/threonine phosphatase (protein phosphatase 6 (PP6)) is a molecular target for GBM radiosensitization treatment. The GBM orthotopic xenograft mice model was used in this study. Our data demonstrated that the protein level of PP6 catalytic subunit (PP6C) was upregulated in the GBM tissue from about $50 \%$ patients compared with the surrounding tissue or control tissue. Both the in vitro survival fraction of GBM cells and the patient survival time were highly correlated or inversely correlated with PP6c expression $\left(R^{2}=0.755\right.$ and -0.707 , respectively). We also found that siRNA knockdown of PP6c reduced DNA-dependent protein kinase (DNA-PK) activity in three different GBM cell lines, increasing their sensitivity to radiation. In the orthotopic mice model, the overexpression of PP6c in GBM U87 cells attenuated the effect of radiation treatment, and reduced the survival time of mice compared with the control mice, while the PP6c knockingdown improved the effect of radiation treatment, and increased the survival time of mice. These findings demonstrate that PP6 regulates the sensitivity of GBM cells to radiation, and suggest small molecules disrupting or inhibiting PP6 association with DNA-PK is a potential radiosensitizer for GBM.

Cell Death and Disease (2011) 2, e241; doi:10.1038/cddis.2011.126; published online 8 December 2011

Subject Category: Cancer

Glioblastoma multiforme (GBM) is one of the most difficult primary cancers to treat and usually rapidly fatal. Owing to the unique structure and function of the brain, radiation therapy is a conventional treatment for the GBM patient along with surgery. Although radiation doubles the survival time of GBM patients, GBM cells are highly resistant to radiation and the tumors are almost never completely eradicated by it. Therefore, increasing the radiation sensitivity of GBM cells is a promising approach to improve GBM patients' survival.

It is well known that ionizing radiation (IR) induces DNA double-strand breaks (DSBs), which is the most severe type of DNA damage. The predominant mechanism of DSB repair is non-homologous end-joining, in which DNA-dependent protein kinase (DNA-PK) plays a central role. DNA-PK is composed of a catalytic subunit (DNA-PKcs) and two Ku heterodimers, which act as regulatory subunits. ${ }^{1}$ It has been shown that DNA-PKcs ${ }^{-1-}$ mice are hypersensitive to IR and that high levels of unrepaired DSBs were observed in DNA$\mathrm{PKcs}^{-1-}$ mice after exposure to other forms of genotoxic agents. ${ }^{2}$ Numerous studies including ours have shown that the DNA-PK activity was increased in radiation-resistant GBM cells. ${ }^{3}$ Chemical inhibitors of DNA-PK, such as NU7441 and
LY294006, show strong sensitization of several tumor cell lines to radiation. ${ }^{4-6}$ These observations suggest that molecules that inhibit DNA-PK activity may be able to improve the outcome of radiation therapy. However, DNA-PKcs is a ubiquitously expressed protein; there is no significant difference in protein level between normal tissue and tumor based on the data from TCGA and our group (data not shown). Therefore, DNA-PKcs itself is not a good molecule target for radiosensitization treatment, as the drugs targeting tumor cells also damage normal tissue, and result in side effects. We have previously found the protein phosphatase 6 (PP6) regulates DNA-PK activity in GBM cells. ${ }^{7}$ If PP6 is differentially expressed in radiation-resistant GBM cells compared with radiation-sensitive GBM cells, PP6 could be a potential target for radiosensitization treatment.

PP6 is a Ser/Thr protein phosphatase classified as a type $2 \mathrm{~A}$ phosphatase family member based on its sequence homology to the catalytic subunit of PP2A. ${ }^{8}$ PP6 is sensitive to active site inhibitors such as okadaic acid, microcystin and calyculin A. ${ }^{9}$ The holoenzyme of PP6 is functionally distinct from other type $2 \mathrm{~A}$ phosphatases and conserved in evolution. ${ }^{10}$ The holoenzyme of PP6 is proposed to be a

\footnotetext{
${ }^{1}$ Key Laboratory of Cell Differentiation and Apoptosis of Chinese Ministry of Education, Institute of Medical Science, Shanghai JiaoTong University School of Medicine, Shanghai, China; ${ }^{2}$ Department of Radiation Oncology, UCLA, Los Angeles, CA, USA; ${ }^{3}$ Renji Hospital, Shanghai Jiao Tong University School of Medicine, Shanghai, China and ${ }^{4}$ Department of Radiation Oncology, University of Virginia, Charlottesville, VA, USA

*Corresponding authors: Y Qiu, Renji Hospital, Shanghai Jiao Tong University School of Medicine, 1630 Dongfang Road, Shanghai 200127, China. Tel: + 86 21 58752345; Fax: + 8621 58394262; E-mail: qiuzhoub@126.com

or J Mi, Key Laboratory of Cell Differentiation and Apoptosis of Chinese Ministry of Education, Institute of Medical Science, Shanghai Jiao Tong University School of Medicine, 7 West Building, Room 306, 280 South Chongqing Road, Shanghai 200025, China. Tel: + 8621 63846590; Fax: + 8621 54660873; E-mail: jmei@sjtu.edu.cn

${ }^{5}$ These authors contributed equally to this work.

Keywords: PP6; GBM; radiation resistance; DNA-PK

Abbreviations: GBM, glioblastoma multiforme; IR, ionizing radiation; DSBs, DNA double-strand breaks; DNA-PK, DNA-dependent protein kinase; DNA-PKcs, DNA-PK catalytic subunit; ATM, ataxia telangiectasia mutated; PP6, protein phosphatase 6; PP6c, PP6 catalytic subunit; RPA32, replication protein A 32-kDa subunit; HGF, hepatocyte growth factor; FBS, fetal bovine serum; MEM, minimum essential medium; DMEM, Dulbecco's modified Eagle's medium

Received 08.9.11; revised 26.10.11; accepted 08.11.11; Edited by P Salomoni
} 
heterotrimer that consists of a catalytic subunit (PP6c), a Sit4associated protein subunit and an ankyrin repeat subunit (ARS). PP6 plays a role in the regulation of NFkB signaling ${ }^{11}$ and IR-induced phosphorylation of histone H2AX. ${ }^{12}$ Our recently published study along with the study from another group have shown that PP6 is a component of the DNA-PK complex; siRNA knockdown of either PP6R1 or PP6c reduces IR-induced DNA-PK activation and increases the sensitivity of glioblastoma M059K cells to radiation. ${ }^{7,12}$ However, it is not clear as to whether PP6 is a potential therapeutic target for radiosensitization of GBM cells in vivo.

In this study, we show that PP6c is upregulated in the glioblastoma (grade IV) tissues of $44.7 \%$ patients, and PP6c protein levels correlate with patient survival time. The lentivirus-mediated PP6c overexpression in GBM U87 cells attenuated the effect of radiation treatment, and reduced the mouse survival time compared to control mice in the orthotopic mice model. While, knocking-down PP6c improved the effect of radiation treatment and increased the mouse survival time. These observations suggest that PP6 might be a therapeutic target for radiosensitization of tumor cells in GBM patients and that PP6 protein level could be a potential biomarker to predict GBM prognosis.

\section{Results}

PP6c expression is increased in glioblastoma tissue from patients and its protein level is inversely correlated with patient survival time. To determine if the PP6c protein is differentially upregulated in glioblastoma tissue and its correlationship with patient survival time, the immunofluorescence staining on the cryosections of glioblastoma tissues was performed and the patient survival time was recorded. In all, 38 patients were recruited in this study.

In total, 10 consecutive sections of glioblastoma tissue were analyzed for each patient. All staining procedures and the microscopy settings were identically performed according to the protocol. Representative PP6c images among sequential imaging from the consecutive sections were selected for further semiquantification analysis by the densitometry. Regarding the subtotal surgical removal and the unique structure of brain, non-tumor glial tissue from epilepsy patients was taken as an additional control in this study besides para-tumor tissues. The fluorescence density of PP6c in both the non-tumor glial tissue from epilepsy patient and the tumor-surrounding tissue was undetectable as a background (Figure 1a), which indicates that the PP6c expression level is low in normal glial cells. However, the GBM sections from about half of patients (17/38; 44.7\%) showed increased PP6c protein expression compared with non-tumor glial tissue or tumor-surrounding tissue. In fact, the average fluorescence density of PP6c in the GBM sections was $50 \%$ brighter than that in non-tumor glial sections or tumor-surrounding tissue. Moreover, according to the microarray data from GBM patients in the Cancer Genomics Center of the NIH (https://www.oncomine.org/resource/main.html), in which the expression profile of PP6c in 237 tissue samples was compared with that in the normal brain tissue, the expression of PP6c is upregulated in GBM tissues compared with normal glial tissue (Figure $1 \mathrm{~b} ; P<0.05$ ). This result is consistent with our immunostaining data (Figure 1a) and data from another group. ${ }^{13}$ DNA-PKcs protein levels were also analyzed in these tissues, and no significant changes in DNAPKcs expression was observed between GBM tissue and normal tissue (data not shown).

On the basis of the ratio of the fluorescence density of PP6c staining in the GBM samples to that in the control tissue, the GBM samples were classified into four groups: the (+ ) group means the fluorescence density increase of PP6c staining is $<20 \%$; the $(++)$ group means this increase is $\geq 20 \%$ and $<40 \%$; the $(+++)$ group means the increase is $\geq 40 \%$ and $<60 \%$; and the $(++++)$ group means the increase is $>60 \%$. The rows from the 3rd to the 6 th of Figure 1a show representative pictures of tissue sections at each level of fluorescence density. According to our analysis, the $65.8 \%$ of patients $(25 / 38)$ were classified into $(++)$ or $(+++)$ groups, only the $10 \%$ of patients (4/38) have strong PP6c staining and the other $9 \%$ patients have weak or low PP6c staining. On the basis of the above semiquantitative classification of PP6c protein levels, the correlations between PP6c protein levels and patient survival time were analyzed. As shown in Figure 1c, patient survival time was highly inversely correlated with PP6c protein levels $\left(R^{2}=0.707, P<0.001\right)$. Patients with high PP6c protein expression tends to have shorter survival time. Moreover, although the patient age is differed from 20 to 65 years old, the data did not show any correlation between age and survival time $\left(R^{2}=0.0078, P=0.5959\right)$. This observation suggests that the overexpression of PP6c in glioblastoma tissues can be taken as a biomarker for prognosis.

PP6c protein level correlates with radiation resistance of glioblastoma cells. To further determine whether PP6 is a potential target for radiosensitization, $10 \mathrm{GBM}$ cell lines, including U87, U251, A172, U138, U118, T98G, M059K, Ln229, Ln-18 and M059J, were used to determine the correlation between PP6c expression levels and radiation resistance. PP6c expression level was detected in these 10 GBM cell lines by real-time PCR. The PP6c expression level was normalized to that of GAPDH. The survival fractions after radiation treatment were measured by the clonogenic assay. The data showed that PP6c expression levels were highly correlated with the survival fraction of glioblastoma cells with $5 \mathrm{~Gy}$ radiation treatment, and the correlation coefficient $\left(R^{2}\right)$ is 0.755 (Figure 2a).

T98G, A172 and U251 were three representative glioblastoma cell lines with significant difference in radiosensitivity, which were selected from the 10 GBM cell lines for further study. Figure $2 \mathrm{~b}$ shows western blots of these three cell lines $1 \mathrm{~h}$ after treatment with or without radiation (5Gy). The protein levels of PP6c were analyzed using densitometry (Image $\mathrm{J}$ software, $\mathrm{NIH}$, Bethesda, MD, USA), and the data were normalized to the density of Ku80 and $\beta$-tubulin. Semiquantification of the immunoblots showed that the protein levels of PP6c were highest in T98G cells, followed by A172 and U251 cells (Figure 2c). Interestingly, DNA-PKcs was found to be cleaved in radiation-treated U251 cells, which were most sensitive to radiation among these 10 GBM cell lines (Figure 2b); however, the total protein level of DNA-PKcs 
a

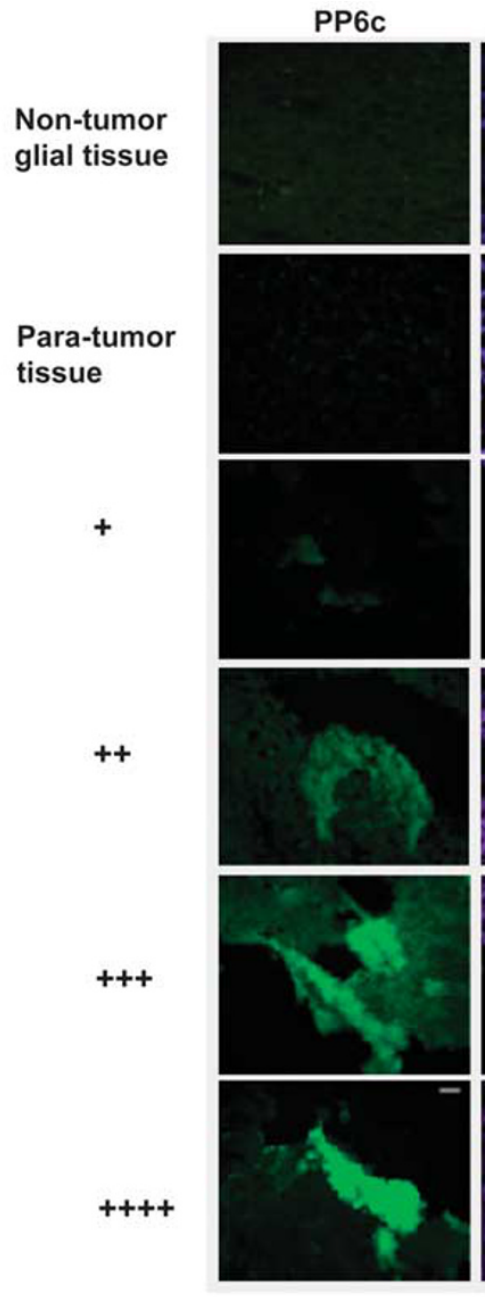

b

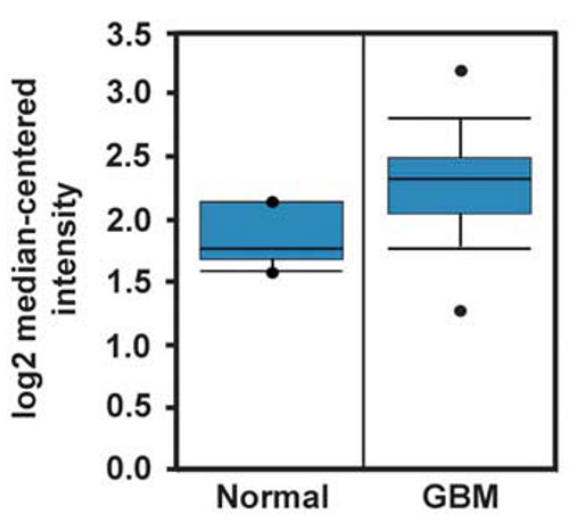

DAPI
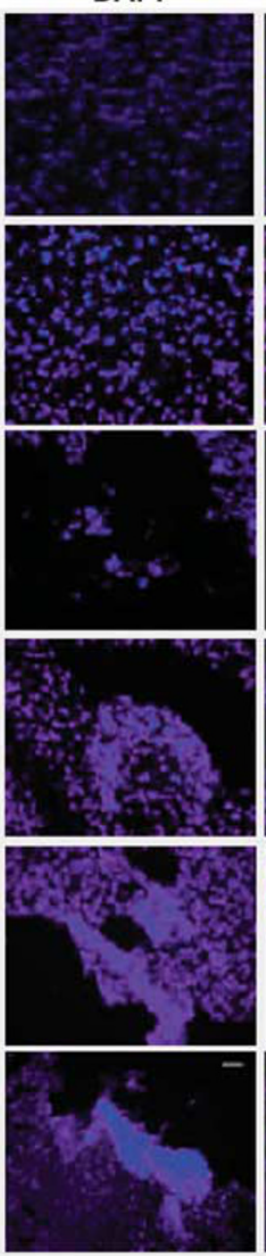

Merge
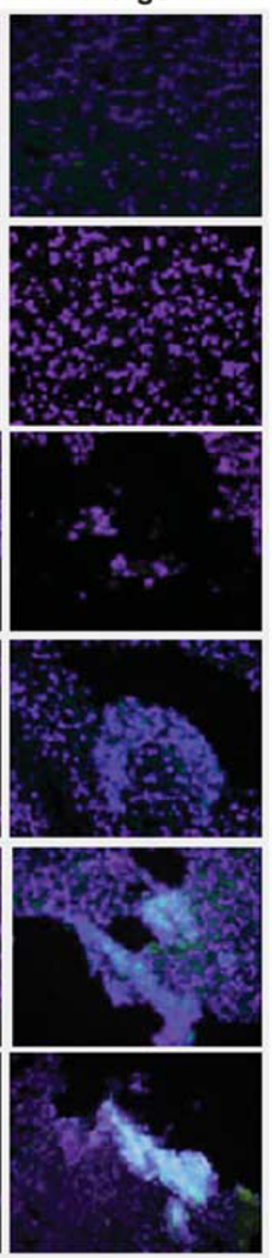

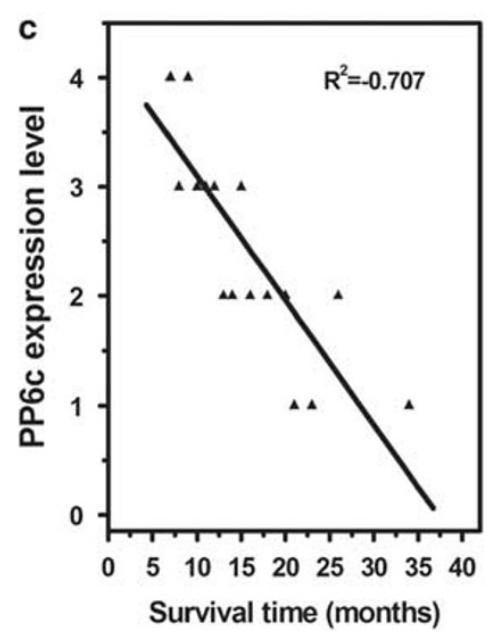

Figure 1 The expression of PP6 catalytic subunit (PP6c) is increased in glioblastoma tissues from patients and its protein levels inversely correlate with patient survival time. (a) The 380 sections from 38 glioblastoma multiforme (GBM) patients (10 slides each patient) were analyzed. The figure rows show representative pictures of non-tumor epilepsy brain section (the 1st row), para-tumor tissue (the 2nd row) and GBM sections (3rd-6th rows). The left panels show PP6c staining (green), the middle panels show 4',6-diamidino-2-phenylindole (DAPI) staining (blue) and the right panels show merged images. The quantification analysis of PP6c fluorescence density (FD) was carried out. The patient samples were classified into four groups ranging from low to high expression $(+, \mathrm{FD}<20 \% ;++, 20 \% \leq \mathrm{FD}<40 \% ;+++, 40 \% \leq \mathrm{FD}<60 \% ;++++$, FD $\geq 60 \%$ ); the scale bar indicates $2 \mathrm{~nm}$. (b) The expression microarray data of GBM tissue downloaded from the public database of the Cancer Genomic Center (TCGA, NIH) was statistically analyzed by Student's $t$-test. The bar represents $\log _{2}$ copy number units of normal brain tissue or GBM tissue (median \pm S.D.). (c) The dose-effect relationship between PP6c protein levels and the survival time (months) of GBM patients was analyzed by the Mann-Whitney U-test. The coefficient of determination $\left(R^{2}\right)$ in the linear regression model is indicated 


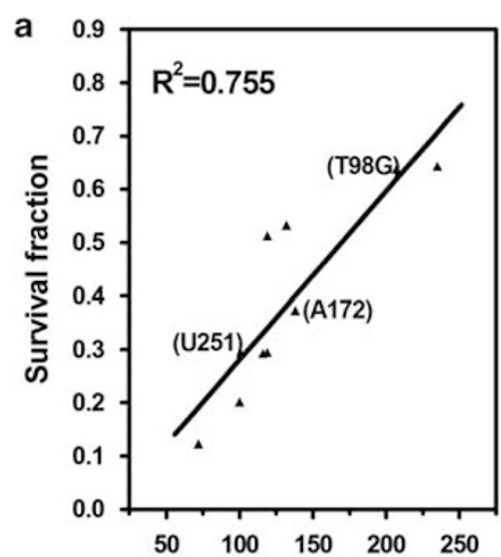

Relative PP6c protein level (\%)

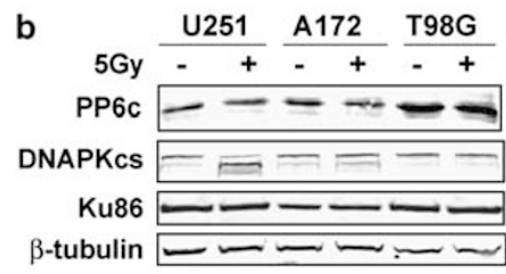

C

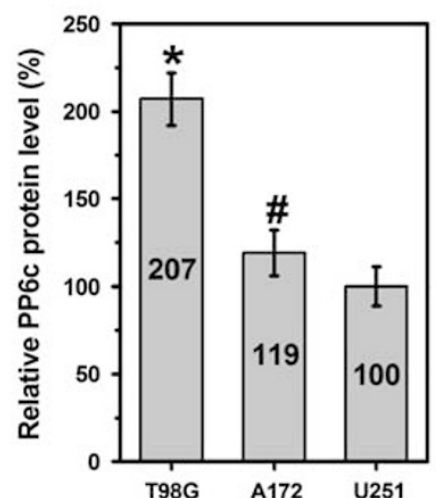

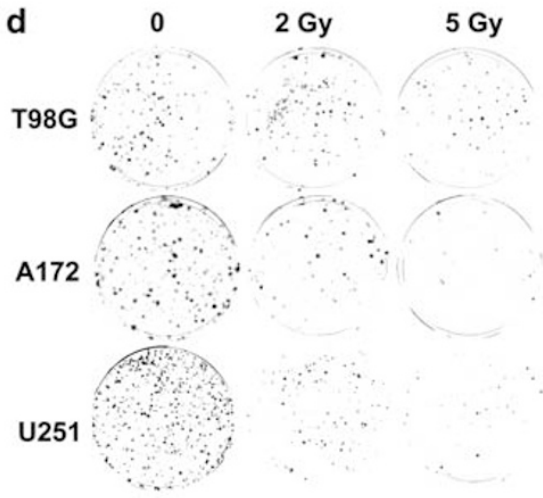

e

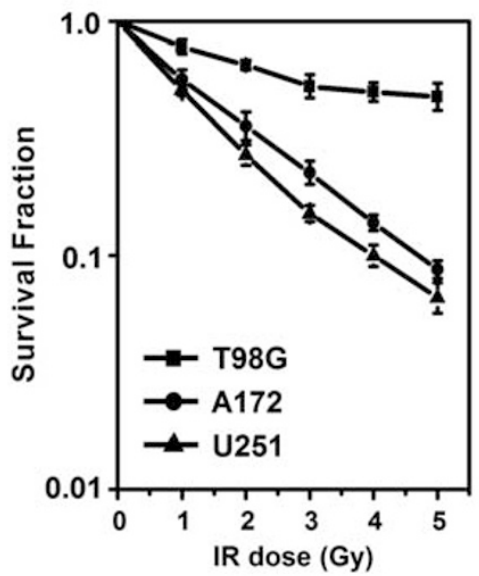

Figure 2 PP6 catalytic subunit (PP6c) protein levels correlate with radiation resistance of glioblastoma cells. (a) The dose-response relationship between PP6 protein levels and the radiosensitivity of glioblastoma cells was analyzed by the Mann-Whitney $U$-test. The coefficient of determination $\left(R^{2}\right)$ in the linear regression model is indicated. (b) T98G, A172 and U251 cells were left untreated or irradiated with 5 Gy. At $1 \mathrm{~h}$ after irradiation, cells were lysed and subjected to immunoblot analysis. The protein levels of DNA-PK catalytic subunit (DNA-PKcs) and PP6c in these cells were detected. (c) The protein levels of PP6c were quantified by densitometry analysis using the Image $\mathrm{J}$ software and the reading was normalized to internal controls of Ku86 and $\beta$-tubulin. The error bars represent the S.D. of the means of three independent experiments. The statistical significance of the differences of the PP6c amount between the different cell lines was determined by Student's $t$-test $\left({ }^{*} P<0.001\right.$; $\left.{ }^{*} P<0.05\right)$. (d) The representative images from clonogenic assay. (e) In all, 100 T98G, A172 or U251 cells treated with the indicated doses of radiation were seeded in a 100 mm dish. Colony numbers were normalized to the untreated controls of each cell line. The error bars represent the S.D. of the means of three independent experiments

was not found to be dramatically increased, which was consistent with the previous microarray data.

In addition, the radiosensitivity of these cells was determined by the clonogenic assays. ${ }^{14}$ Approximately $60 \%$ of T98G cells survived after irradiation with $5 \mathrm{~Gy}$ compared to only $8.86 \%$ of $A 172$ cells and $6.87 \%$ of $U 251$ cells (Figure $2 d$ ). The plating efficiencies were about $40 \%$ without radiation treatment in these experiments. Apparently, the T98G cells were most resistant to radiation among the three glioblastoma cell lines, which was consistent with the data from another group. ${ }^{3}$ These observations suggest a relationship between PP6c expression levels and radiation resistance.
IR-induced DNA-PK activity is correlated with radiation resistance in glioblastoma cells. To determine whether DNA-PK activity has a dose-effect relationship with the radiosensitivity of glioblastoma cells, the three representative GBM cell lines, T98G, A172 and U251, were used for this study. The DNA-PK activities in three cell lines were measured $30 \mathrm{~min}$ post-IR, with or without Nu7026, a DNAPK-specific inhibitor. As shown in Figure 3a, IR-induced DNA-PK activity was 2.3 times higher in T98G cells than in A172 cells, and 3 times higher than in U251 cells. Nu7026 abrogated the effect of IR on DNA-PK activity, which is close to the level of that in the untreated cells. The correlation 

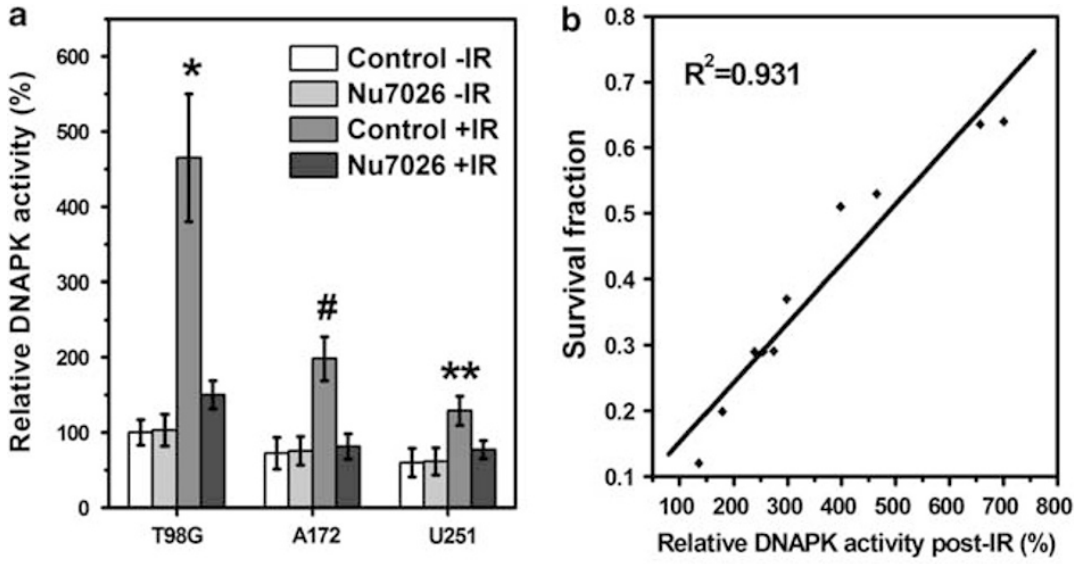

C

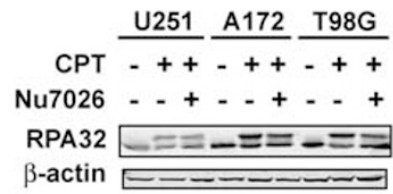

d

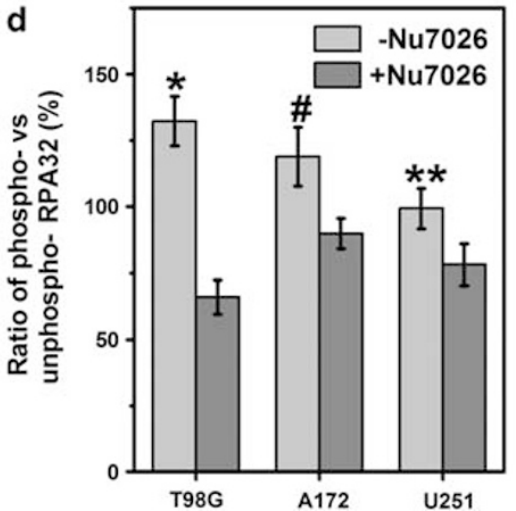

Figure 3 lonizing radiation (IR)-induced DNA-dependent protein kinase (DNA-PK) activity is correlated with radiation resistance in glioblastoma cells. (a) T98G, A172 and U251 cells were treated with Nu7026 $(10 \mu \mathrm{M}) 30 \mathrm{~min}$ before irradiation ( 5 Gy) or without radiation. At $1 \mathrm{~h}$ post-irradiation, the nuclear fractions from these cells were isolated, and then subjected to a DNA-PK kinase assay using a specific peptide as substrate $\left({ }^{*} P<0.001 ;{ }^{\#, * \star} P<0.05\right)$. (b) The dose-response relationship between DNA-PK activity and the survival fraction of glioblastoma cells was analyzed by the Mann-Whitney $U$-test. The coefficient of determination $\left(R^{2}\right)$ in the linear regression model is indicated. (c) T98G, A172 and U251 cells were treated with Nu7026 $(10 \mu \mathrm{M})$ for $30 \mathrm{~min}$ before camptothecin (CPT) treatment. At $3 \mathrm{~h}$ post-irradiation, whole-cell lysates were subjected to immunoblotting for total or phospho-specific RPA32 detection using RPA32 antibody (top row). $\beta$-Actin was used as a loading control. (d) Phosphorylated and unphosphorylated RPA32 were quantified by the Image $\mathrm{J}$ software, and the readings were normalized to the internal control of $\beta$-tubulin. The error bars represent the S.D. of the means of three independent experiments. The statistical significance of the differences between the ratio of phosphorylated to unphosphorylated RPA32 in each cell line was determined by Student's $t$-test $\left({ }^{*} P<0.001 ;{ }^{\#, * \star} P<0.05\right)$

between DNA-PK activity and radiation resistance in T98G, A172 and U251 cells was determined by the Mann-Whitney $U$-test. Similar to PP6c protein levels, DNA-PK activity was highly correlated $\left(R^{2}=0.931\right)$ with the survival fraction of the glioblastoma cells (Figure $3 b$ ).

Moreover, the endogenous DNA-PK activity was also measured through detecting the phosphorylation of endogenous replication protein $A$, whose $32-k D a$ subunit (RPA32) is differentially phosphorylated by three phosphatidylinositol-3kinases (ataxia telangiectasia mutated (ATM), ATM-Rad3 related, DNA-PK) in response to different DNA-damaging agents. DNA-PK is the primary kinase responsible for RPA32 phosphorylation in response to camptothecin (CPT) treatment, ${ }^{15-17}$ and thus CPT-induced phosphorylation of RPA32 is an indirect measure of DNA-PK activity. Phosphorylation of RPA32 is observed in immunoblots as the appearance of a band of reduced mobility relative to the parent RPA32 band, and this new band can be detected by an RPA32 Ser 4/Ser 8 phospho-specific antibody. CPT treatment induced RPA32 phosphorylation in all three glioblastoma cell lines (Figure 3c), and the DNA-PK specific inhibitor Nu7026 attenuated this phosphorylation (upper bands) of the endogenous RPA32 (Figure $3 \mathrm{c}$ ). On the basis of our calculation, the ratio of phosphorylated RPA32 to unphosphorylated RPA32 in the radiation-treated T98G cells was 2 times more than that in IR plus Nu7026-treated T98G cells, which in A172 cells or U251 cells was 1.32 or 1.26 times, respectively (Figure $3 d$ ). Using statistical correlationship analysis, the change in CPTstimulated phosphorylation of RPA32 was highly correlated $\left(R^{2}=0.931\right)$ with the survival fraction of the glioblastoma cells (data not shown). These data further demonstrated the DNAPK activity was distinct in the different GBM cells.

Taken together, these observations suggest a strong relationship between DNA-PK activity and radiation resistance.

siRNA knockdown of PP6c reduces DNA-PK activity and increases the sensitivity of glioblastoma cells to radiation. To further verify whether PP6 regulates radiosensitivity of GBM cells through DNA-PK, it was necessary to assess the effects of PP6 on DNA-PK activity and radiosensitivity in these cells. To address this question, 
we transfected our three glioblastoma cell lines, T98G, A172 and U251, with siRNA to specifically knock down PP6c. The activity of DNA-PK was analyzed in nuclear extracts using an in vitro kinase assay with a specific peptide substrate. In the absence of IR treatment, siRNA knockdown of PP6c only slightly affected the basal activity of DNA-PK (Figure 4a; light gray bars versus empty bars). The knocking-down efficiency of PP6c was determined by western blot (Figure 4a). However, post-IR DNA-PK activity in all three cell lines increased 2- to 4.5-fold over basal activity levels, while the
siRNA knockdown of PP6c in all three cell lines markedly reduced the IR-induced DNA-PK activity under similar conditions (Figure 4a; dark gray bars versus middle gray bars).

In the mean time, the PP6c were stably overexpressed in U251 cells or stably knocked down in T98G cells by the lentivirus-mediated infection, as the endogenous PP6c protein level in the U251 cells was much lower than that in the T98G cells. After the CPT treatment, the CPT-induced RPA32 phosphorylation in PP6c downregulated T98G cells
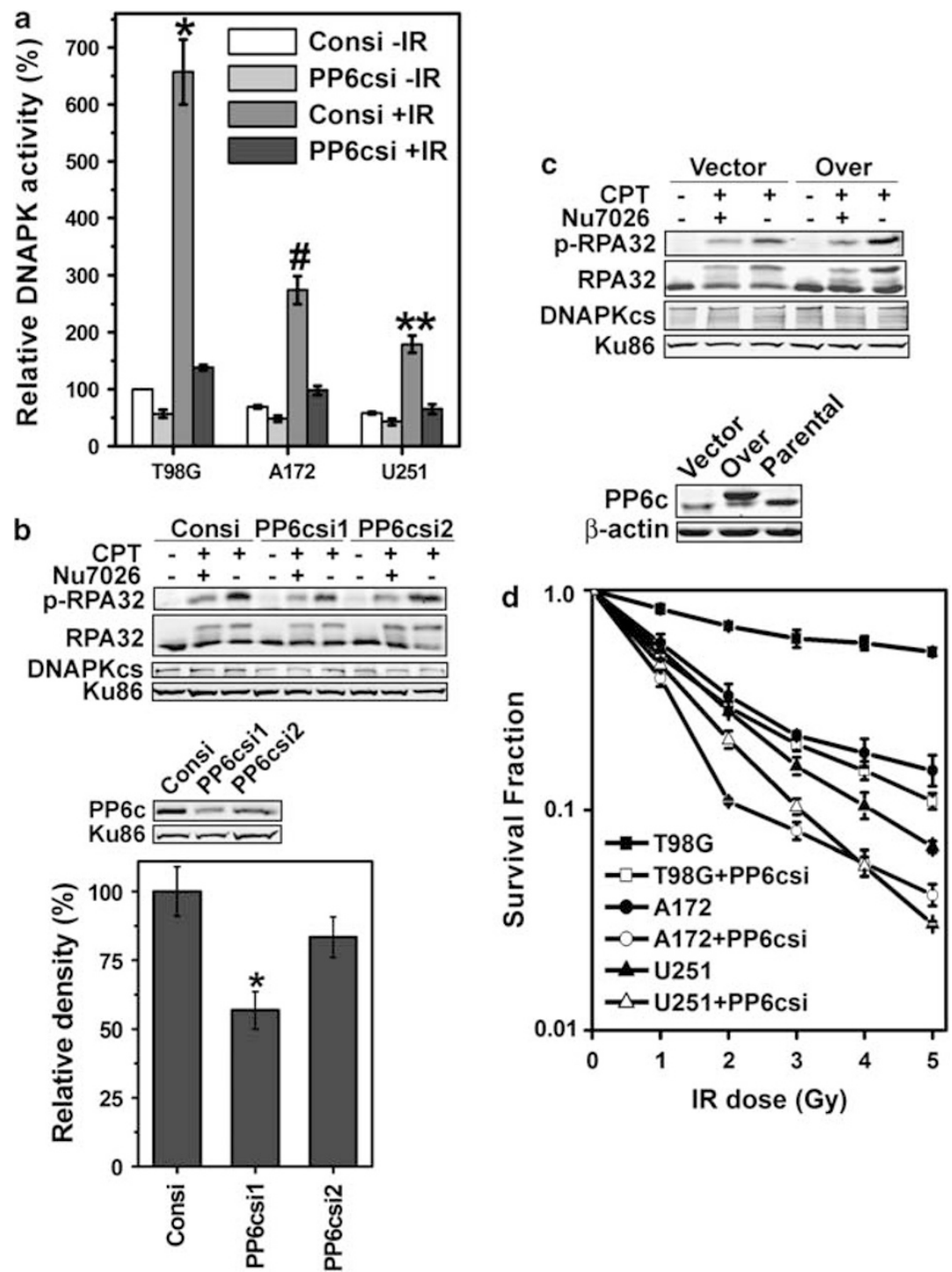

Figure 4 Small interfering RNA (siRNA) knockdown of PP6 catalytic subunit (PP6c) reduces DNA-dependent protein kinase (DNA-PK) kinase activity in three different glioblastoma cell line and increase the sensitivity of glioblastoma cells to radiation. (a) T98G, A172 and U251 cells were transfected with PP6c-specific siRNA or non-specific siRNA. At $48 \mathrm{~h}$ after transfection, the cells were treated with $5 \mathrm{~Gy}$ radiation or sham radiation, and subjected to the DNA-PK kinase assay using a specific peptide as substrate. ${ }^{30,31}$ The data were normalized to the number of non-irradiated and non-PP6c siRNA-transfected T98G cells. The error bars represent the S.D. of the means of three independent experiments. The statistical significance of the differences between the DNA-PK activity in the irradiated PP6c siRNA-treated or control siRNA-treated cells was determined by Student's $t$-test $\left(^{*, *, * \star} P<0.001\right)$. The western blotting showed the siRNA knocking-down efficiency of PP6c in T98G, A172 and U251 cells. (b) The T98G cells infected by the lentivirus containing PP6c siRNAs or control siRNA were treated with camptothecin (CPT)- and/or DNA-PK-specific inhibitor Nu7026, the Ser 4/8 phosphorylation of RPA32 were detected by western blot and the bottom panel shows PP6c knocking-down efficiency in stable T98G cells. (c) The U251 cells infected by the lentivirus containing PP6c coding sequence or control siRNA were treated with CPT- and/or DNA-PK-specific inhibitor Nu7026, the Ser 4/8 phosphorylation of RPA32 were detected by western blot and the bottom panel shows PP6c protein level in stable U251 cells. (d) T98G, A172 and U251 cells were transfected with PP6c-specific siRNA or nonspecific siRNA and then treated with the indicated dose of radiation. All colony numbers were normalized to the untreated control of each cell line. The error bars represent the S.D. of the means of three independent experiments 
were significantly lower than that in control T98G cells (Figure 4b), while the CPT-induced RPA32 phosphorylation in PP6c overexpressed U251 cells were obviously higher than that in control U251 cells (Figure 4c). These observations indicated that the DNA-PK activity, at least the CPT-induced DNA-PK activation, was regulated by PP6. These data suggest that the DNA-PK activity in radiation-resistant glioblastoma cells can be modulated through the regulation of PP6, and either the downregulation of PP6c or the inhibition of DNA-PK activity can improve the radiation resistance of these cells.

To further test whether PP6 regulates the radiosensitivity of these three glioblastoma cells, clonogenic assays were performed in T98G, A172 and U251 cells transfected with PP6c-specific siRNA or control siRNA. The plating efficiencies were $20-30 \%$ after transfection of siRNA in these experiments. The siRNA knockdown of PP6c significantly increased the sensitivity of these glioblastoma cells to radiation compared with the nonspecific siRNA-transfected cells (Figure $4 d$; open symbols versus filled symbols). These observations demonstrated that PP6 does regulate the GBM radiation sensitivity, and suggest that PP6 is a candidate molecular target for GBM radiosensitization.

PP6c knocking-down improves the radiation therapeutic efficacy on the mice bearing orthotopic xenografted glioblastoma. The glioblastoma U87 cells overexpressing PP6c or knocking-down PP6c were intracranially injected into the right caudate nucleus of the athymic nude mice $\left(1 \times 10^{6}\right.$ each), with the aid of a small animal stereotactic frame, and 72 mice were injected. The U87 cells infected with empty viruses were taken as control. At 10 days after injection, mice bearing tumor (62 mice) were randomly divided into two groups according to the MRI imaging, including IR and non-IR irradiation. Each group contains control, overexpression and knock-down subgroup (10 mice in each subgroup). The $6 \mathrm{~Gy}$ of total radiation were administered over three times in 1 week, and the schedule was described in Materials and methods. The survival time of each mouse was recorded, and all survival times in each group were analyzed using Kaplan-Meier method. Our results showed that the radiation treatment prolonged the mice survival time in all group. With radiation treatment, the mice in the PP6c knocking-down group survived longest (53 days, median survival time) among the mice in three groups; the mice survival time in the control group and the PP6c overexpression group were 42.5 and 32.5 days, respectively (Figure 5a). Although without radiation treatment the mice survival time in the PP6c knocking-down group was longer than that in other groups too, the radiation enhancement of survival time in the PP6c knocking-down group was $26.9 \%$, much higher than that in the control group and the PP6c overexpression group, which was 14.2 and $7.3 \%$, respectively. The differences were found to be statistically significant $(P<0.001)$.

On the basis of the MRI image of tumors at the 9th day after intracranial injection, the tumor size in each group had some but not significant difference (representative pictures shown in the top row of Figure $5 \mathrm{~b}$ ). After radiation treatment, the tumor size in the PP6c knocking-down group at the 21st day did not show significant growth compared to the tumor size of the same mouse at the 9th day (representative pictures shown in the right panels of Figure $5 \mathrm{~b}$ ). As same as our expectation, the tumor sizes in the PP6c overexpression group at the 21st day were obviously larger than the tumor size of the same mouse at the 9th day (representative images as shown in the middle panels of Figure $5 b$ ). The average volumes of tumor in each group have been shown in the graph of Figure $5 \mathrm{~b}$; the tumor sizes shrank more (54.3\%) in the PP6c knocking-down group than that in the control group (38.9\%) and PP6c overexpressing group (16.3\%), although the tumor volumes are different in each group. The fluorescence immunostaining of PP6c protein level in GBM tissue were shown in Figure 5c.

\section{Discussion}

There is ample evidence in the literature that cellular phosphatase activity and radiosensitivity are functionally related. Nakamura et al. ${ }^{18}$ reported that the serine/threonine protein phosphatase inhibitor calyculin A enhanced cell killing when cells were treated $30 \mathrm{~min}$ before X-ray irradiation. Recently, Chapman's group reported that HT-29 human colon carcinoma cells treated with inhibitors of protein phosphatases 1 and 2 were significantly more sensitive to radiation than untreated cells. ${ }^{19,20}$ However, these chemicals are highly cytotoxic to all cells, including normal cells, and how to increase the sensitivity of tumor cells to radiation while minimizing the side effects caused by a radiosensitizing drug is still challenging to radiobiologists. Owing to the unique structure and function of the brain, subtotal surgery, one of two major conventional treatments, cannot completely remove GBM cells - even when combined with radiotherapy, the efficacy of which is never satisfactory owing to the high resistance of GBM cells to radiation. The deficiency of these methods results in worse prognosis of GBM patients. Thus, finding a way to enhance the efficacy of radiotherapy is critical to the survival of GBM patients.

Our data demonstrated that PP6 regulated IR-induced DNAPK activation, and the DNA-PK activity as well as the PP6C protein levels inversely correlated with the radiosensitivity in GBM cells. Interestingly, the protein level of DNA-PKcs was not upregulated in the cells with high expression of PP6c based on the western blot and immunofluorescence staining. These observations indicate that PP6 regulates the radiosensitivity of GBM cells by modulating DNA-PK activity rather than increasing DNA-PKcs protein expression. The importance of DNA-PK in radiosensitivity regulation and DNA repair is well known. ${ }^{1,2,21-23}$ Although several chemical compounds directly targeting DNA-PK activity as a radiosensitizer are currently under development, exploring a more specific molecular target in GBM cells is necessary for more efficacy and less side effects because of the importance of DNA-PK function in normal cells. In this study, our in vitro and in vivo data suggest that PP6c is a promising biomarker for GBM prognosis and may play a critical role in the progression of GBM, although the mechanism by which PP6c expression is upregulated in GBM cells is still unknown. Moreover, we noted that the PP6c is not equally expressed in all GBM tissues, even in the same GBM tissue, which suggests that these GBM cells 
a

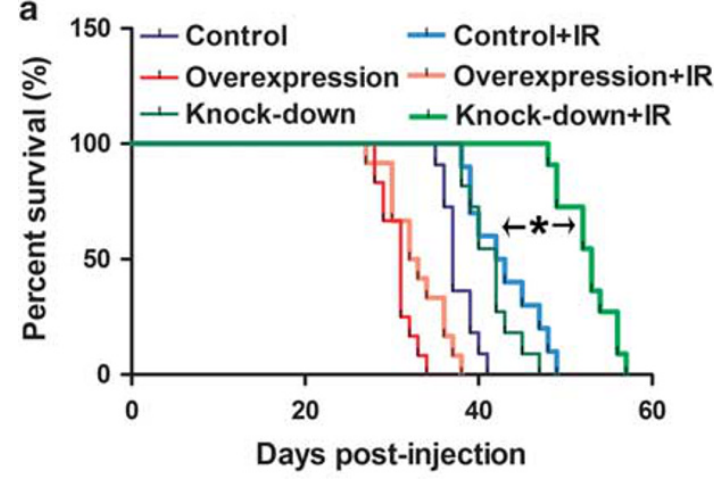

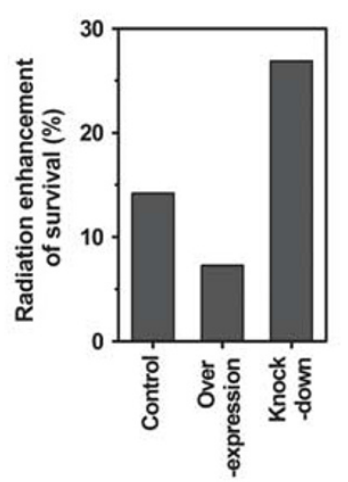

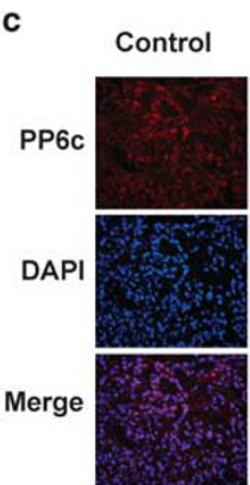

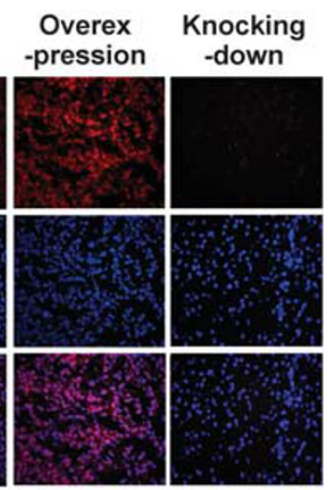

b

$-I R$

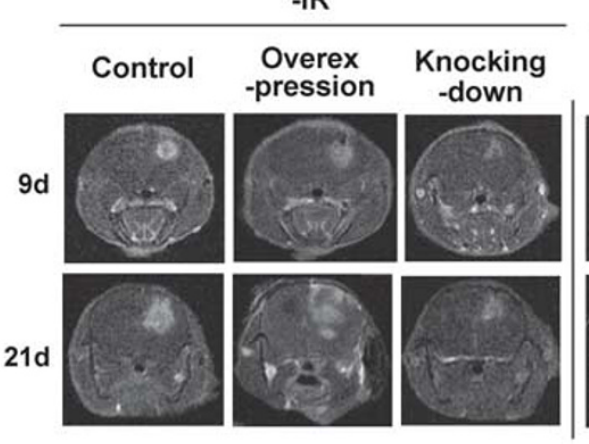

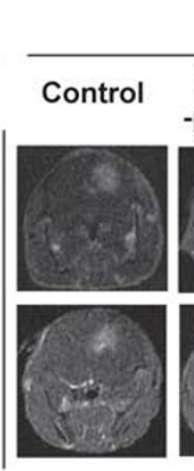

$+\mid \mathrm{R}$

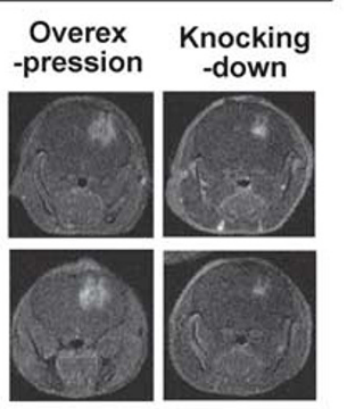

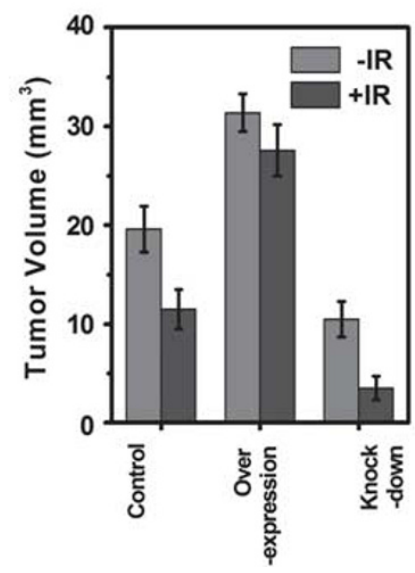

Figure 5 Small interfering (siRNA) knockdown of PP6 catalytic subunit (PP6c) improves the radiation therapeutic efficacy on the mice bearing orthotopic xenografted glioblastoma. (a) The mice were euthanized at the time of reaching a moribund condition, and all survival times in each group were analyzed using Kaplan-Meier method. This experiment was independently repeated two times, six mice in each group at the start time point. The analyzed mice with radiation treatment in empty virus group (control), PP6c knocking-down or PP6c overexpression group were 10,11,12, respectively, while the mice without radiation treatment in the control group, PP6c knocking-down or PP6c overexpression group were 11,11, 12, respectively. The radiation enhancements of survival time in each group were calculated by the formula: the survival time with radiation divided by the survival time without radiation. The significance of radiation enhancement difference between control siRNA group and PP6c siRNA group were analyzed by the $\chi^{2}$ method ( $\left.{ }^{*} P<0.001\right)$. (b) The representative tumor pictures from the PP6c overexpression, the PP6c knocking-down or the control group were captured by magnetic resonance imaging (MRI) imaging at the 9th and 21st day with (+ IR (ionizing radiation)) or without radiation treatment (-IR), and the tumor volumes were calculated by the formula: $\frac{1}{2} \times L \times S^{2}$ ( $L$ : maximum length; $S$ : minimum length). (c) The representative PP6c immunofluorescence staining (red) in glioblastoma sections from the PP6c overexpression, the PP6c knocking-down or the control group

overexpressing PP6c acquire the extra capability of radiation resistance.

Furthermore, our in vivo data have shown that the radiation enhancement of survival time in the PP6c knocking-down groups are highest (26.9\%) compared with the PP6c overexpression group (7.3\%) and the control group (14.2\%); the difference between the control group and either PP6c knocking-down group or PP6c overexpression group has statistical significance $(P<0.001)$. The previous study shows that DNA-PKcs is inactivated by phosphorylation at Thr 3950, and thus PP6c overexpression possibly enhances the IRinduced DNA-PK activation through dephosphorylation of Thr $3950,{ }^{24}$ which is one possible mechanism by which PP6c knockdown increases radiation sensitivity of GBM cells. In addition, PP6 is involved in a diversity of cellular function, including gene transcription, cell-cycle progression and apoptosis, ${ }^{25,26}$ all of which would influence tumor growth, and PP6c knockdown may affect tumor growth through regulating these progresses. These observations suggest that PP6 not only regulates the sensitivity of GBM cells to radiation, but also affects the glioblastoma growth without radiation, and both effects will benefit the GBM patients.

This study opens up new avenues for radiosensitization in that molecules inhibiting PP6 activity or disrupting PP6 association with DNA-PK may improve the sensitivity of GBM cells to radiation, although the safety of PP6 inhibitor or molecules disrupting PP6/DNA-PK association has not been tested as yet. Hepatocyte growth factor (HGF)/Met receptor is another important protein kinase involved in radiation resistance and cell proliferation. The Wang group reported that Met protected endothelial cells against radiation-induced damage, ${ }^{27}$ and PP2A, a PP6 family member, is a critical protein phosphatase regulating Met activity through phosphorylation of Ser985. ${ }^{28}$ It is unknown if PP6 regulates GBM radiosensitivity through the HGF receptor as DNA-PK may not be the only downstream target for PP6-regulated radiosensitization. Owing to the large number of growth factor signaling pathways PP6 participates in, we cannot at this point exclude other mechanisms mediating PP6-regulated radiosensitization.

In summary, we conclude that small molecules selectively inhibiting PP6 activity are possible drugs for radiosensitization 
treatment. ${ }^{9}$ In addition, according to the observation that DNA-PK mediates PP6c's regulation of radiosensitivity, small molecules disrupting the association of PP6 with DNA-PK might be able to reduce selectively the DNA repair response to $I R$ and may thus be useful in enhancing the efficacy of radiotherapy.

\section{Materials and Methods}

Patients and eligibility. This study was approved by the Ethical Review Board of the Medical Faculty of the Shanghai Jiao-Tong University School of Medicine. Patients from 20 to 65 years of age with newly diagnosed, histologically confirmed GBM (World Health Organization grade IV astrocytoma) in the temporal or frontal lobe were eligible for this study. In total, 38 patients were recruited in this study. The patients were diagnosed and treated in the Shanghai Renji Hospital between January 2006 and December 2007. These eligible patients received standard radiotherapy (fractionated focal irradiation in daily fractions of 2 Gy given 5 days per week for 6 weeks) plus temozolomide $\left(150 \mathrm{mg} \mathrm{per}^{2}\right.$ of body-surface area per day, 5 days per week) within 1 month after surgery for subtotal removal. The primary end point was patient death.

Cell lines and reagents. Glioblastoma T98G, U138 and U87 cells were maintained in minimum essential medium (MEM; Invitrogen, Carlsbad, CA, USA) supplemented with 10\% fetal bovine serum (FBS; Invitrogen); U118, Ln-229, Ln-18, A172 and U251 glioblastoma cells were maintained in Dulbecco's modified Eagle's medium (DMEM; Invitrogen) supplemented with 10\% FBS (Invitrogen); and M059K and M059J glioblastoma cells were maintained in DMEM/F12 (1:1) (Invitrogen) supplemented with $10 \% \mathrm{FBS}$ (Invitrogen). All cells were maintained at $37^{\circ} \mathrm{C}$ with $5 \%$ $\mathrm{CO}_{2}$ and were in an exponential growth phase at the time of irradiation. The following commercial antibodies were used: anti-DNA-PKcs pan-mouse monoclonal, anti-Ku86 mouse monoclonal and anti- $\beta$-tubulin mouse monoclonal (Santa Cruz Biotechnology, Santa Cruz, CA, USA); anti-PP6c rabbit polyclonal (Bethyl Laboratories, Montgomery, TX, USA); anti-RPA32 mouse monoclonal and $\beta$-actin mouse monoclonal (Sigma, St Louis, MO, USA), Alex 488-conjugated antirabbit and Alex 594-conjugated anti-mouse secondary antibodies (Invitrogen) and Vectashield Mounting Medium with DAPI (Vector Laboratories, Burlingame, CA, USA). The DNA-PK kinase assay kit was obtained from Promega Inc. (Madison, WI, USA). All other reagents were purchased from Sigma.

The fluorescent immunostaining of GBM blocks from patients or mice model. Dissected glioblastoma blocks were fixed with $4 \%$ paraformaldehyde and embedded in paraffin, and 10 slices were generated from each block. Non-tumor glial tissue from epilepsy patients or tumor-surrounding tissue was used as a control. Followed by deparaffinization and hydration, the paraffin-embedded sections were subjected to immunostaining and visualized by fluorescence microscopy (Leica DM 2500, Shanghai, China). All staining procedures and the microscopy settings were identically performed according to the protocol. Images were captured and analyzed by the Openlab software (Openlab, Shanghai, China).

The orthotopic GBM mice model. Congenitally athymic nude mice (athymic Ncr-nu/nu), 5-6 weeks old (Charles River Laboratories, Wilmington, MA, USA), were used in this study. The orthotopic tumor model for glioblastoma on a protocol was approved by the Shanghai Jiao-Tong University Animal Care and Use Committee. To prepare cells for transfer to the intracranial compartment, the glioblastoma U87 cells either overexpressing or knocking-down PP6c, which were validated by previous studies, or U87 cells infected with empty lentiviruses were exponentially growing in culture dishes.

The cells at $90 \%$ confluent were trypsinized, and re-suspended in phosphatebuffered saline (PBS). A cell suspension containing $1 \times 10^{6}$ cells per $5 \mu$ I PBS was used for one implantation into the striatal region of the athymic mouse brain. Under deep isoflurane anesthesia, mice were placed in a small-animal stereotactic frame (David Kopf Instrument, Tujunga, CA, USA). A sagittal incision was made through the skin to expose the cranium, and a burr hole was made in the skull at $0.2 \mathrm{~mm}$ anterior and $1.8 \mathrm{~mm}$ lateral (right) from the bregma using a small dental drill. At a depth of $3 \mathrm{~mm}$ from the brain surface, $5 \mu \mathrm{l}$ of cell suspension was injected. After $5 \mathrm{~min}$, the needle was removed and the hole was sealed with bone wax (W810;
Johnson \& Johnson International, Somerville, NJ, USA). The wound was sutured immediately.

The mice were observed for body weight loss. They were killed by cervical dislocation when they presented signs of neurological disorders or when their body weight loss was $>20 \%$ of their original weight, the time to this moment since surgery is considered as 'survival time'. Their brains were embedded into OCT medium (Leica Inc., Wetzlar, Germany) and quickly frozen in $-80^{\circ} \mathrm{C}$ after resection. The GBM cryosections (CM1850; Leica Inc.) were processed for histopathological analysis and fluorescence immunostaining of Alex 594-labeled PP6c. The intragroup variation in the length of survival included standard deviation and $95 \%$ confidence interval determinations were analyzed.

In vivo MRI imaging of tumor. GBM-bearing mice with or without radiation were examined on day 9,16 and 21 days post-tumor implantation to detect the growth of the grafted tumor fragments. Tumor imaging in orthotopic mice model was performed with a small animal coil on a High-Field GE Signa 3-Tesla clinical MR scanner (General Electric, Waukesha, WI, USA), and images were obtained by using a standard T1 protocol following gadolinium (Gd-DTPA, $100 \mu / 20 \mathrm{~g}$; Bayer Healthcare Pharmaceuticals, Montville, NJ, USA) intraperitoneal injection $10 \mathrm{~min}$ before examination. In enhanced scanning, scanning parameters was AxT1 FSE series scan plane: oblique; FOV: 5.0 ; phase FOV: 0.60 ; slice thickness: $1.0 \mathrm{~mm}$; spacing: $0.0 \mathrm{~mm}$; freq DIR: R/L; auto-TR: 600; and minimum TR: 60 . Tumor sizes were measured, and their volumes were calculated according to the following formula: $\left(\right.$ length $\times$ width $\left.^{2}\right) / 2$ using the Function Analysis software (General Electric); these results were subsequently analyzed using the Image Pro-plus software (General Electric). ${ }^{29}$

Overexpression or siRNA knockdown of PP6c. Exponentially growing T98G, A172 or U251 cells were infected with lentiviruses containing the coding DNA sequence of PP6c (NM_001123355) or the specific siRNA (50 nM) against PP6c siRNA1: 5'-AGACAGATAACACAGGTCT-3'; siRNA2: TCGATCATGGTCTTCAA $A G$. The preparation of lentiviruses were according to the protocol as described previously. ${ }^{7}$ A nonspecific siRNA (sequence: $5^{\prime}$-AAAUCUUCGAGACAUUCUGUU- $3^{\prime}$ ) was used as a control. The other siRNA oligonucleotides used in this study were purchased from Dharmacon (Lafayette, CO, USA), and transfected into the cells using Lipofectamine RNAiMAX (Invitrogen) according to the manufacturer's instructions.

Radiation treatment. Cells in culture were irradiated with an irradiator (GE 3000 ) using a ${ }^{137} \mathrm{Cs}$ source at a dose rate of $4.0 \mathrm{~Gy} / \mathrm{min}$. During irradiation, the cultures were maintained in a container designed to mimic the conditions of the cell culture incubator $\left(5 \% \mathrm{CO}_{2}\right.$ and $95 \%$ air at $\left.37^{\circ} \mathrm{C}\right)$. For the $\mathrm{GBM}$ mice model, mice were observed daily following tumor cell injection; 10 days after xenograft transplantation, the healthy mice were randomized to radiation treatment group of 12 mice each. Radiation was delivered to the entire head of unanesthetized mice at 2 Gy each time. The mice were immobilized in a plastic restraint, through a single right lateral beam from a ${ }^{137} \mathrm{Cs}$ source. The remainder of the body was shielded with a lead block. The radiation schedule was used during the course of this study: $2 \mathrm{~Gy}$ $\mathrm{M}-\mathrm{W}$ - $\mathrm{F}$ for 1 week ( $6 \mathrm{~Gy}$ in total) as established through preliminary studies. All mice used for therapy response evaluations were euthanized at the time of reaching a moribund condition.

Immunoprecipitation. Exponentially growing T98G, A172 or U251 cells were irradiated with $10 \mathrm{~Gy} I \mathrm{R}$, then harvested at indicated time points and finally lysed in $1 \mathrm{ml}$ of lysis buffer (0.5\% (v/v) NP-40, $5 \mathrm{mM}$ EDTA, $2 \mathrm{mM}$ EGTA, $20 \mathrm{mM}$ MOPS, $1 \mathrm{mM}$ PMSF, $20 \mathrm{mM}$ sodium pyrophosphate, $30 \mathrm{mM}$ sodium fluoride, $40 \mathrm{mM}$ $\beta$-glycerophosphatase, $1 \mathrm{mM} \mathrm{Na}_{3} \mathrm{VO}_{3}$ and protease inhibitors) with caspase inhibitor Z-VAD-FMK. Aliquots of $1 \mathrm{mg}$ total protein were mixed with $4 \mu \mathrm{g}$ of monoclonal anti-DNA-PKcs antibody at $4{ }^{\circ} \mathrm{C}$ overnight. The bound proteins were recovered by binding to $25 \mu$ of protein-A agarose (Sigma). The samples were separated by $7.5 \%$ SDS-PAGE gel electrophoresis, then transferred to nitrocellulose overnight and separated by SDS-PAGE and finally transferred to nitrocellulose membranes. Proteins of interest were detected with specific antibodies, followed with infrared dye 680- or 800-conjugated secondary antibodies. Blots were scanned using an Odyssey infrared imaging system (Licor, Lincoln, NE, USA), and proteins were quantitatively analyzed by the Odyssey software (Licor).

DNA-PK kinase assay. Exponentially growing T98G, A172 or U251 cells were treated with $10 \mathrm{~Gy} I R$. After $30 \mathrm{~min}$, the nuclear extracts were prepared as described previously. ${ }^{7}$ Briefly, the cells were permeabilized, and the nuclei were 
extracted in lysis buffer containing $0.42 \mathrm{M} \mathrm{NaCl}$ and $1.5 \mathrm{mM} \mathrm{MgCl}$. DNA-PK activity was analyzed using a DNA-PK activity assay kit ${ }^{30,31}$ according to the manufacturer's instructions (Promega Inc.).

Clonogenic assay. The clonogenic assay was adapted from Franken et al. ${ }^{14}$ Briefly, the IR-treated and -untreated control cells were harvested and 400 cells per dish re-seeded in a $100 \mathrm{~mm}$ dish at an appropriate density to obtain approximately 50-100 colonies. Following 10-14 days of incubation, cells were fixed and stained with crystal violet, and colonies containing at least 50 cells were scored.

Statistical analysis. The Kaplan-Meier estimator (GraphPad Prism 5, GraphPad Software, La Jolla, CA, USA) ${ }^{32}$ was used to generate the survival curves and to estimate the median survival values. Differences between survival curves were compared using a log-rank test. ${ }^{33}$ Two-way categorical comparisons (i.e., PP6c overexpression versus radiation treatment sensitivity, PP6c knocking-down versus radiation treatment sensitivity) were carried out using Fisher's exact test. Correlationship of PP6C protein levels with patient survival time or cell survival fraction, and correlationship between DNA-PK activity and cell survival fraction were analyzed by the use of Spearson analysis (two-tailed) (GraphPad Prism, GraphPad Software). We also used the Student's $t$-test and Mann-Whitney U-test when appropriate. All tests were twosided and $P<0.05$ was considered to be statistically significant.

\section{Conflict of Interest}

The authors declare no conflict of interest.

Acknowledgements. This work was supported by the Shanghai Education Committee via a grant support provided by the National Program on Key Basic Research Project (973 Program) (2012CB967000) and the National Science Foundation of China fund (81072076) to Dr. JM, the National Science Foundation of China fund (31000349) to Dr. YS, the Science and Technology Commission of Shanghai Municipality (0952nm03900) and Key discipline project of Renji Hospital (RJ4101307) to Dr. YQ and by the Science and Technology Commission of Shanghai Municipality to Dr. XF (09zr1417700).

1. Lees-Miller SP. The DNA-dependent protein kinase, DNA-PK: 10 years and no ends in sight. Biochem Cell Biol 1996; 74: 503-512.

2. Collis SJ, DeWeese TL, Jeggo PA, Parker AR. The life and death of DNA-PK. Oncogene 2005; 24: 949-961.

3. Hosoi $\mathrm{Y}$, Miyachi H, Matsumoto $\mathrm{Y}$, Ikehata H, Komura J, Ishii K et al. A phosphatidylinositol 3-kinase inhibitor wortmannin induces radioresistant DNA synthesis and sensitizes cells to bleomycin and ionizing radiation. Int J Cancer 1998; 78: 642-647.

4. Zhao Y, Thomas HD, Batey MA, Cowell IG, Richardson CJ, Griffin RJ et al. Preclinical evaluation of a potent novel DNA-dependent protein kinase inhibitor NU7441. Cancer Res 2006; 66: 5354-5362.

5. Shinohara ET, Geng L, Tan J, Chen H, Shir Y, Edwards E et al. DNA-dependent protein kinase is a molecular target for the development of noncytotoxic radiation-sensitizing drugs. Cancer Res 2005; 65: 4987-4992.

6. Fuhrman CB, Kilgore J, LaCoursiere YD, Lee CM, Milash BA, Soisson AP et al. Radiosensitization of cervical cancer cells via double-strand DNA break repair inhibition. Gynecol Oncol 2008; 110: 93-98.

7. Mi J, Dziegielewski J, Bolesta E, Brautigan DL, Larner JM. Activation of DNA-PK by ionizing radiation is mediated by protein phosphatase 6. PLoS One 2009; 4: e4395.

8. Honkanen RE, Golden T. Regulators of serine/threonine protein phosphatases at the dawn of a clinical era? Curr Med Chem 2002; 9: 2055-2075.

9. Prickett TD, Brautigan DL. Overlapping binding sites in protein phosphatase $2 A$ for association with regulatory A and alpha-4 (mTap42) subunits. J Biol Chem 2004; 279 : 38912-38920.

10. Clotet J, Gari E, Aldea M, Arino J. The yeast ser/thr phosphatases sit4 and ppz1 play opposite roles in regulation of the cell cycle. Mol Cell Biol 1999; 19: 2408-2415.

11. Kajino $T$, Ren $H$, lemura $S$, Natsume $T$, Stefansson $B$, Brautigan $D L$ et al. Protein phosphatase 6 down-regulates TAK1 kinase activation in the IL-1 signaling pathway. J Biol Chem 2006; 281: 39891-39896.

12. Douglas $P$, Zhong J, Ye R, Moorhead GB, Xu X, Lees-Miller SP. Protein phosphatase 6 interacts with the DNA-dependent protein kinase catalytic subunit (DNA-PKcs) and dephosphorylates \{gamma\}-H2AX. Mol Cell Biol 2010; 30: 1368-1381.
13. Zhong J, Liao J, Liu X, Wang P, Liu J, Hou W et al. Protein phosphatase PP6 is required for homology-directed repair of DNA double-strand breaks. Cell Cycle 10: 1411-1419.

14. Franken NA, Rodermond HM, Stap J, Haveman J, van Bree C. Clonogenic assay of cells in vitro. Nat Protoc 2006; 1: 2315-2319.

15. Shao RG, Cao CX, Zhang $H$, Kohn KW, Wold MS, Pommier $Y$, Replicationmediated DNA damage by camptothecin induces phosphorylation of RPA by DNAdependent protein kinase and dissociates RPA:DNA-PK complexes. EMBO J 1999; 18 1397-1406.

16. Block WD, Yu Y, Lees-Miller SP. Phosphatidyl inositol 3-kinase-like serine/threonine protein kinases (PIKKs) are required for DNA damage-induced phosphorylation of the $32 \mathrm{kDa}$ subunit of replication protein A at threonine 21. Nucleic Acids Res 2004; 32 : 997-1005.

17. Sakasai R, Shinohe K, Ichijima Y, Okita N, Shibata A, Asahina K et al. Differential involvement of phosphatidylinositol 3-kinase-related protein kinases in hyperphosphorylation of replication protein A2 in response to replication-mediated DNA double-strand breaks. Genes Cells 2006; 11: 237-246.

18. Nakamura K, Antoku S. Enhancement of X-ray cell killing in cultured mammalian cells by the protein phosphatase inhibitor calyculin A. Cancer Res 1994; 54: 2088-2090.

19. Biade S, Stobbe CC, Boyd JT, Chapman JD. Chemical agents that promote chromatin compaction radiosensitize tumour cells. Int J Radiat Biol 2001; 77: 1033-1042.

20. Price WA, Stobbe CC, Park SJ, Chapman JD. Radiosensitization of tumour cells by cantharidin and some analogues. Int J Radiat Biol 2004; 80: 269-279.

21. Abraham RT. PI 3-kinase related kinases: 'big' players in stress-induced signaling pathways. DNA Repair (Amst) 2004; 3: 883-887.

22. Anderson CW, Lees-Miller SP. The nuclear serine/threonine protein kinase DNA-PK. Crit Rev Eukaryot Gene Expr 1992; 2: 283-314.

23. Goodarzi AA, Yu Y, Riballo E, Douglas $P$, Walker $S A, Y e R$ et al. DNA-PK autophosphorylation facilitates Artemis endonuclease activity. $E M B O J 2006 ; 25$ : 3880-3889.

24. Douglas P, Cui X, Block WD, Yu Y, Gupta S, Ding $Q$ et al. The DNAdependent protein kinase catalytic subunit is phosphorylated in vivo on threonine 3950, a highly conserved amino acid in the protein kinase domain. Mol Cell Biol 2007; 27: $1581-1591$.

25. Stefansson $B$, Brautigan $D L$. Protein phosphatase PP6 $N$ terminal domain restricts $G 1$ to $S$ phase progression in human cancer cells. Cell Cycle 2007; 6: 1386-1392.

26. Douglas P, Zhong J, Ye R, Moorhead GB, Xu X, Lees-Miller SP. Protein phosphatase 6 interacts with the DNA-dependent protein kinase catalytic subunit and dephosphorylates gamma-H2AX. Mol Cell Biol 2010; 30: 1368-1381.

27. Hu SY, Duan HF, Li QF, Yang YF, Chen JL, Wang LS et al. Hepatocyte growth factor protects endothelial cells against gamma ray irradiation-induced damage. Acta Pharmacol Sin 2009; 30: 1415-1420.

28. Hashigasako A, Machide M, Nakamura T, Matsumoto K. Bi-directional regulation of Ser-985 phosphorylation of $\mathrm{c}$-met via protein kinase $\mathrm{C}$ and protein phosphatase $2 \mathrm{~A}$ involves c-Met activation and cellular responsiveness to hepatocyte growth factor. J Biol Chem 2004; 279: 26445-26452.

29. Lu CC, Lin HF, Lee HS, Kao WY. Metastatic colon cancer presenting as Pancoast's disease. Am J Surg 2009; 197: e51-e52.

30. Ding Q, Reddy YV, Wang W, Woods T, Douglas P, Ramsden DA et al. Autophosphorylation of the catalytic subunit of the DNA-dependent protein kinase is required for efficient end processing during DNA double-strand break repair. $\mathrm{Mol}$ Cell Biol 2003; 23: 5836-5848.

31. Reddy YV, Ding Q, Lees-Miller SP, Meek K, Ramsden DA. Non-homologous end joining requires that the DNA-PK complex undergo an autophosphorylation-dependent rearrangement at DNA ends. J Biol Chem 2004; 279: 39408-39413.

32. Molins CR, Delorey MJ, Yockey BM, Young JW, Sheldon SW, Reese SM et al. Virulence differences among Francisella tularensis subsp. tularensis clades in mice. PLoS One 2010; 5: e10205

33. Sarkaria JN, Carlson BL, Schroeder MA, Grogan P, Brown PD, Giannini C et al. Use of an orthotopic xenograft model for assessing the effect of epidermal growth factor receptor amplification on glioblastoma radiation response. Clin Cancer Res 2006; 12 (Part 1): 2264-2271.
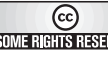

Cell Death and Disease is an open-access journal published by Nature Publishing Group. This work is licensed under the Creative Commons Attribution-Noncommercial-Share Alike 3.0 Unported License. To view a copy of this license, visit http:// creativecommons.org/licenses/by-nc-sa/3.0/ 